Slavica

bruxellensia

\section{Slavica bruxellensia}

Revue polyphonique de littérature, culture et histoire

slaves

$8 \mid 2012$

Migration(s) et Exil(s)

\title{
Alain Blum \& Yuri Shapoval, Faux coupables - surveillance, aveux et procès en Ukraine soviétique (1924-1934)
}

\section{Brice Thissen}

\section{(2) OpenEdition}

Journals

Édition électronique

URL : http://journals.openedition.org/slavica/1059

DOI : 10.4000/slavica.1059

ISSN : 2034-6395

Éditeur

Université libre de Bruxelles - ULB

Référence électronique

Brice Thissen, « Alain Blum \& Yuri Shapoval, Faux coupables - surveillance, aveux et procès en Ukraine soviétique (1924-1934) », Slavica bruxellensia [En ligne], 8 | 2012, mis en ligne le 25 juin 2012, consulté le 21 septembre 2020. URL : http://journals.openedition.org/slavica/1059 ; DOI : https://doi.org/ 10.4000/slavica.1059

Ce document a été généré automatiquement le 21 septembre 2020.

\section{c) (i) $\Theta$}

Les contenus de Slavica bruxellensia sont mis à disposition selon les termes de la Licence Creative Commons Attribution - Pas d'Utilisation Commerciale - Pas de Modification 3.0 France. 


\section{Alain Blum \& Yuri Shapoval, Faux coupables - surveillance, aveux et procès en Ukraine soviétique (1924-1934)}

Brice Thissen

\section{RÉFÉRENCE}

Blum A. \& Shapoval Y., Faux coupables - surveillance, aveux et procès en Ukraine soviétique (1924-1934) - l'exemple de M. Gruševskij et S. Efremov, CNRS Editions, Collections « Mondes russes et est-européens. Etats, Sociétés, Nations », Paris, 2012, 350 p. 
1 Le propos principal de cet ouvrage est d'étudier la logique des modes de surveillance utilisés en URSS dans les années 1920 et 1930. À travers ce prisme, les auteurs veulent présenter la mise en place $\mathrm{du}$ pouvoir stalinien comme un processus complexe et non-uniforme menant à la répression jusqu'à l'absurde. Ils n'oublient pas de replacer ces méthodes dans leur contexte historique et politique, c'est pourquoi ils étudient les méthodes de surveillance en parallèle avec les fonctions et les conséquences des répressions et des procès, auxquels elles mènent directement, qui jalonnent la montée au pouvoir de Staline.

Le second propos développé est celui des relations entre la Russie soviétique et l'Ukraine. L'objectif de cet ouvrage est de

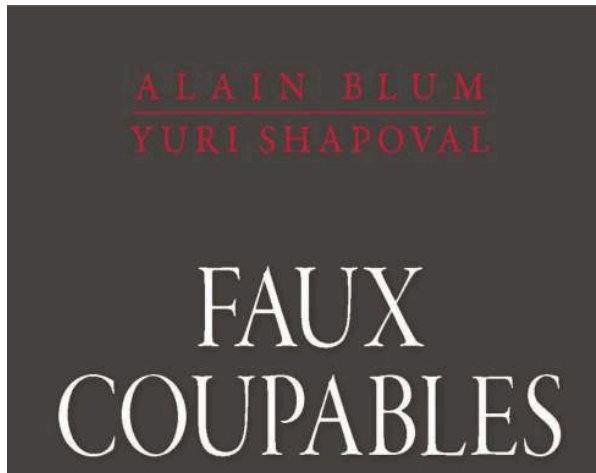
reconstituer devant le lecteur les étapes

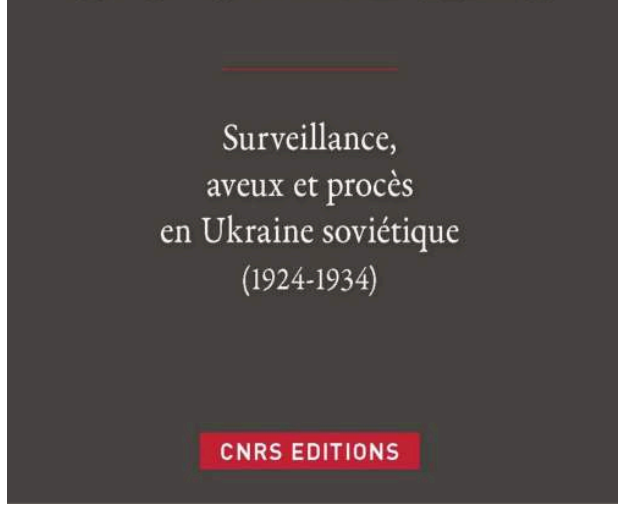
du développement de la grille de lecture utilisée par les soviétiques pour diriger leurs vagues de répression, mais aussi d'en exposer l'application en évoquant deux figures importantes de l'intelligentsia ukrainienne, Mikhail S. Gruševskij (1866-1934) et Sergej A. Efremov (1876-1939). Ces deux cas sont étudiés sur la base du recoupement de sources d'origine diverses: journaux intimes, aveux, dépositions etc. (tous ces documents sont accessibles au lecteur soit à la fin du livre, soit sur la page internet http://cercec.ehess.fr/ fauxcoupables).

3 L'ouvrage est divisé en cinq chapitres précédés d'une introduction. Il possède aussi le récapitulatif des acronymes des partis politiques citées et leurs descriptions, ainsi qu'un index de noms. Efremov y est présenté en tant que savant, spécialiste de littérature et publiciste. Il était en outre l'un des fondateurs du Parti démocratique ukrainien (1905). Condamné en 1930 à dix ans de privation de liberté, il décéda en réclusion en mars 1939. Gruševskij est quant à lui identifié en tant qu'historien et président du Conseil (Rada) centrale d'Ukraine (1917-1918). A partir de 1929, il fut membre de l'Académie des sciences d'URSS, responsable du secteur histoire de l'Ukraine. Ces savants emblématiques des sciences sociales et humaines ukrainiennes, avaient tous deux participé activement au premier gouvernement ukrainien, avant d'être séparés par le destin. Faux coupables est ainsi également une histoire des hommes qui se jalousent et haïssent malgré une estime intellectuelle réciproque. Les biographies des deux personnages mentionnés illustrent en effet très bien les relations entre les mondes académique et politique et témoignent bien des tensions qui agitent ceux-ci à partir de la fin de la Première Guerre Mondiale. Leurs comportements vis-àvis du pouvoir soviétique et les motifs qui les animent sont abordés et développés sur la base des écrits, des lettres ou des extraits des journaux intimes des intéressés. Les auteurs dressent également un tableau général de la politique menée par le pouvoir 
soviétique vis-à-vis des milieux intellectuels en émigration et des élites du pays au début des années 1920.

4 Après la lecture du second chapitre, le lecteur comprend ce qui a fait défaut aux élites intellectuelles dans leur lutte contre le pouvoir soviétique, mais il se rend également compte des moyens dont celui-ci disposait pour arriver à ses fins. On y prend également conscience que les soviétiques eux-mêmes, malgré le caractère implacable de leur politique, n'ont pas toujours eu conscience des résultats que leurs décisions engendreraient.

Les erreurs de jugement et d'appréciation commises par les intellectuels sont au centre du troisième chapitre troisième, chapitre crucial car il expose les mécanismes qui ont permis aux soviétiques de fixer une bonne fois pour toute une ligne de conduite dans leurs rapports avec les élites intellectuelles ukrainiennes. Ce chapitre donne au lecteur la pleine conscience de l'importance de l'égarement de l'intelligentsia.

6 Le chapitre suivant montre la dernière étape de reconstitution de la grille de lecture utilisée par les services de sécurité, car les auteurs y esquissent un tableau de l'usage qui sera fait par la suite des renseignements accumulés. La structure des organes de sécurité tels que la GPU, l'OGPU et le NKVD est aussi exposée au lecteur et illustrée par des organigrammes.

7 Le cinquième et dernier chapitre est consacré à la grille de lecture qui a permis au pouvoir soviétique de justifier ses répressions. Cette fois-ci, le but des soviétiques est clairement explicité au lecteur : ils veulent mettre la société en anarchie et disloquer les réseaux sociaux existants pour les remplacer par une relation verticale avec Staline, qui s'impose comme la seule autorité. À l'aune des mêmes exemples, les auteurs reviennent sur les périodes de la vie de Gruševskij et de Efremov tout en déchiffrant leur passé à travers la loupe soviétique. Le schéma que suivent les soviétiques dans leurs actions de répression est expliqué étape par étape, ainsi que les différents moyens de pression exercée sur les personnages cités ci-dessus. Cette façon de procéder permet au lecteur de mesurer les proportions prises par la sphère politique au détriment de la sphère privée.

Celle-ci me paraît très riche et apporte une plus value importante au propos développé par les auteurs parce qu'elle rassemble les documents auxquels les auteurs font allusion au court de la lecture - ce sont les numéros repris entre crochets, mais également une liste des noms des partis cités, un index des personnalités, des affaires criminelles politiques évoquées ainsi qu'une liste des journaux cités. L'autre partie de l'annexe est disponible sur la page internet citée plus haut. Le tout permet au lecteur de s'orienter rapidement et de saisir clairement le sens des propos consignés par les protagonistes dans leurs journaux et lettres. Les plus curieux pourront prendre connaissance de tous les détails qu'ils souhaitent, les autres peuvent se contenter de la seule lecture de l'ouvrage.

Cet ouvrage est très bien construit et les objectifs annoncés dans l'introduction sont, d'après moi, atteints. La diversité des sources est un réel atout, car elle permet de saisir dans son entièreté le décalage entre les réactions de l'intelligentsia et la politique menée par les soviétiques. Pour cette même raison, le lecteur prend conscience que, malgré leur côté écrasant et irrémédiable, les décisions prises par le pouvoir central sont le fruit de tâtonnement et que la politique vis-à-vis des élites culturelles n'a pas été menée de façon uniforme. 
10 De même, les documents rendus accessibles au lecteur à la fin du livre et sur internet permettent de s'immerger dans les faits exposés et rendent la matière accessible à tout un chacun.

11 Pour l'étudiant de slavistique, il n'y a qu'un seul bémol : les relations entre la Russie et l'Ukraine ne sont pas vraiment développées, bien qu'elles soient annoncées dans l'introduction comme un des thèmes abordés. C'est la seule ombre que je vois à ce tableau impressionnant.

INDEX

Mots-clés : élites intellectuelles ukrainiennes, histoire de l'Ukraine, procès politiques, Mikhail S. Gruševskij, Sergej A. Efremov

Index chronologique : communisme, entre-deux-guerres, XXe siècle

Index géographique : Ukraine, URSS

\section{AUTEURS}

\section{BRICE THISSEN}

Diplômé en Langues et Littératures modernes, orientation slaves, Université Libre de Bruxelles 